\title{
Anti-tumour compounds illudin S and Irofulven induce DNA lesions ignored by global repair and exclusively processed by transcription- and replication-coupled repair pathways
}

\author{
Nicolaas G.J. Jaspers ${ }^{\mathrm{a}, *}$, Anja Raams ${ }^{\mathrm{a}}$, Michael J. Kelner ${ }^{\mathrm{b}}$, Jessica M.Y. Nga , \\ Yukiko M. Yamashita ${ }^{\mathrm{c}}$, Shiunichi Takeda ${ }^{\mathrm{c}}$, Trevor C. McMorris ${ }^{\mathrm{b}}$, \\ Jan H.J. Hoeijmakers ${ }^{a}$ \\ ${ }^{a}$ Department of Cell Biology and Genetics, Erasmus Medical Center, P.O. Box 1738, 3000 DR Rotterdam, The Netherlands \\ b Department of Pathology, UCSD Medical Centre, San Diego, CA, USA \\ ${ }^{c}$ Department Radiation Genetics, Graduate School of Medicine, Kyoto University, Kyoto, Japan
}

Received 1 May 2002; received in revised form 12 August 2002; accepted 20 August 2002

\begin{abstract}
Illudin $S$ is a natural sesquiterpene drug with strong anti-tumour activity. Inside cells, unstable active metabolites of illudin cause the formation of as yet poorly characterised DNA lesions. In order to identify factors involved in their repair, we have performed a detailed genetic survey of repair-defective mutants for responses to the drug. We show that $90 \%$ of illudin's lethal effects in human fibroblasts can be prevented by an active nucleotide excision repair (NER) system. Core NER enzymes XPA, XPF, XPG, and TFIIH are essential for recovery. However, the presence of global NER initiators XPC, HR23A/HR23B and XPE is not required, whereas survival, repair and recovery from transcription inhibition critically depend on CSA, CSB and UVS, the factors specific for transcription-coupled NER. Base excision repair and non-homologous end-joining of DNA breaks do not play a major role in the processing of illudin lesions. However, active RAD18 is required for optimal cell survival, indicating that the lesions also block replication forks, eliciting post-replication-repair-like responses. However, the translesion-polymerase DNA pol $\eta$ is not involved.

We conclude that illudin-induced lesions are exceptional in that they appear to be ignored by all of the known global repair systems, and can only be repaired when trapped in stalled replication or transcription complexes. We show that the semisynthetic illudin derivative hydroxymethylacylfulvene (HMAF, Irofulven), currently under clinical trial for anti-tumour therapy, acts via the same mechanism.
\end{abstract}

(C) 2002 Elsevier Science B.V. All rights reserved.

Keywords: Xeroderma pigmentosum; Cockayne syndrome; Ultraviolet; Mushroom toxin; Irofulven; Excision repair; Post-replication repair; Non-homologous end-joining; Tumour therapy

Abbreviations: BER, base excision repair; CS, Cockayne syndrome; GG-NER, global-genome nucleotide excision repair; HMAF, hydroxymethylacylfulvene; NHEJ, non-homologous end-joining; PRR, post-replication repair; TC-NER, transcription-coupled nucleotide excision repair; UDS, unscheduled DNA synthesis; UVS, UV-sensitivity syndrome; XP, xeroderma pigmentosum

* Corresponding author. Fax: +31-10-4089468.

E-mail address: jaspers@gen.fgg.eur.nl (N.G.J. Jaspers). 


\section{Introduction}

The illudins are a family of natural sesquiterpene compounds with anti-tumour activity, originally isolated from the mushrooms Omphalotus illudens (jack-o-lantern) or Lampteromyces japonicus [1]. Although many illudins are highly effective against various drug-resistant tumours both in vivo and in vitro, the extreme cytotoxicity of these compounds in the nanomolar range has severely restricted their practical use in cancer therapy $[2,3]$. Recently semisynthetic derivatives have been reported [4] with a strongly improved therapeutic index $[2,5,6]$ of which hydroxymethylacylfulvene (HMAF, Irofulven) is currently under clinical trial $[7,8]$. The tissue specificity and tumour selectivity of the illudins has been attributed to the presence of an energy-dependent system mediating transport into the cells [9] and subsequent metabolic activation to an unknown reactive intermediate [10]. Inside the cells, strong inhibition of DNA synthesis occurs, presumably caused by DNA damage [2]. The requirement for prolonged incubation periods of actively metabolising cells has hampered the identification of the responsible DNA adduct [11]. Early

Table 1

Properties of cells strains used

\begin{tabular}{|c|c|c|c|c|}
\hline $\begin{array}{l}\text { Human fibroblast } \\
\text { strain }\end{array}$ & Clinical symptoms & Affected gene & NER activity $(\%)^{\mathrm{a}}$ & $\begin{array}{l}\text { Repair pathway } \\
\text { affected }^{b}\end{array}$ \\
\hline C5RO & Normal control & - & 100 (deficient) & - \\
\hline XP25RO & XP with neurological complications & $X P A$ & $<1$ & GG-NER + TC-NER \\
\hline XPCS1BA & $\mathrm{XP} / \mathrm{CS}$ complex & $X P B(E R C C 3)$ & 5 & \\
\hline XP6BE & XP with neurological complications & $X P D(E R C C 2)$ & 25 & \\
\hline XP42RO & Mild XP & $X P F(E R C C 4)$ & $15-30$ & \\
\hline XP2BI & XP & $X P G(E R C C 5)$ & 2 & \\
\hline XP21RO & $\mathrm{XP}$ & & 17 & GG-NER \\
\hline XP35RO & & $X P C$ & 15 & \\
\hline XP4LE & & & 20 & \\
\hline XP2RO & Mild XP & $X P E(D D B 2)$ & 61 & \\
\hline CS3BE & Cockayne syndrome & CSA $(E R C C 8)$ & $\sim 100$ & TC-NER \\
\hline CS1AN & Cockayne syndrome & $C S B(E R C C 6)$ & & \\
\hline Kps3 & UVS syndrome & Not cloned & & \\
\hline XP1RO & Mild XP (variant) & RAD3OA (POLH) & $\sim 100$ & $\begin{array}{l}\text { Translesion } \\
\text { DNA synthesis }\end{array}$ \\
\hline \multicolumn{5}{|l|}{ XP30RO } \\
\hline 94RD103 & Non-photosensitive trichothiodystrophy & Not cloned & $\sim 100$ & $\mathrm{NER}=$ normal \\
\hline Other cell strains & Species (origin) & Affected gene(s) & Repair pathway affected & \\
\hline $43-3 B$ & Chinese hamster ovary (CHO9) & ERCC1 & NER and crosslink repair & \\
\hline EM-9 & Chinese hamster ovary (AA8) & $X R C C 1$ & Base excision repair & \\
\hline V15B & Chinese hamster ovary (V79B) & Ки 86 & Non-homologous end-joining & \\
\hline $\mathrm{XR}-\mathrm{C} 1$ & Chinese hamster ovary (CHO9) & $D N A-P K c s$ & & \\
\hline XR-1 & Chinese hamster ovary (CHO-K1) & $X R C C 4$ & & \\
\hline HR23DKO & $\begin{array}{l}\text { Double-targeted mouse mutant } \\
\text { embryonic fibroblasts }\end{array}$ & $H R 23 A$ and $H R 23 B$ & GG-NER & \\
\hline $\mathrm{XPC}-$ & Targeted mouse embryonic fibroblasts & $X P C$ & & \\
\hline DT40 $(\Delta \operatorname{rad} 18)$ & Chicken lymphoma (DT40) & $R A D 18$ & Post-replication repair & \\
\hline
\end{tabular}

\footnotetext{
${ }^{a}$ Measured as UV-induced unscheduled DNA synthesis (UDS).

${ }^{\mathrm{b}}$ GG, global-genome; TC, transcription-coupled; NER, nucleotide excision repair.
} 
genetic studies using UV-sensitive rodent mutant cell lines have indicated that nucleotide excision repair (NER) is involved in the removal of illudin-induced lesions [11]. However, contribution of other repair pathways such as base excision repair (BER) or recombinational repair cannot be excluded as long as the spectrum of lesions remains undefined.

In order to further investigate the mechanisms of the extreme toxicity of the illudins, we have studied the toxicity and repair of illudin $\mathrm{S}$ lesions in a variety of mammalian cell strains with known defects in DNA repair processes, including NER, BER, non-homologous end-joining (NHEJ) and post-replication repair (PRR), (listed in Table 1). Surprisingly, the data indicate that illudin-induced DNA adducts are ignored by the global-genome repair pathways (NER, BER and NHEJ), and can only be processed when trapped in sites of stalled transcription or replication forks. Transcription-coupled NER and post-replication repair are the key processes protecting cells to illudin $\mathrm{S}$ toxicity.

\section{Materials and methods}

\subsection{Cell strains and culture conditions}

Human fibroblast strains were cultured in Ham F10 supplemented with $15 \%$ foetal bovine serum and antibiotics. For Chinese hamster ovary cells a 1:1 mixture of Ham F10 and DMEM was used. Chicken lymphoma cells were cultured at $39^{\circ} \mathrm{C}$ in HEPES-buffered RPMI1640, supplemented with 10\% inactivated calf serum and $1 \%$ chicken serum. Properties of the strains used in this study are summarised in Table 1.

\subsection{Agents}

UV light of predominantly $254 \mathrm{~nm}$ was emitted from a Philips TUV germicidal tube (fluence rate $\left.0.3 \mathrm{~W} / \mathrm{m}^{2}\right)$. Stock solutions of illudin $\mathrm{S}(0.2 \mu \mathrm{g} / \mathrm{ml}$ corresponding to $0.76 \mu \mathrm{M}$ ), isolated from $O$. illudens as described [12] and HMAF (Irofulven, $1 \mathrm{mg} / \mathrm{ml}$ ) are fully stable at $4{ }^{\circ} \mathrm{C}$ [2] and diluted in culture medium to the desired concentrations, immediately prior to use. Radioactive nucleic acid precursors were all purchased from Amersham Radiochemicals.

\subsection{Cell survival assays}

To avoid the time-consuming and laborious colony assays of primary human fibroblasts, a simplified approach was followed for measurement of cytotoxicity. Sparse cultures $\left(5 \times 10^{3}\right.$ to $10 \times 10^{3}$ cells per $6 \mathrm{~cm}$-dish, or $1 \times 10^{3}$ to $2 \times 10^{3}$ per $3 \mathrm{~cm}$-dish) were grown for $48 \mathrm{~h}$ and then exposed to illudin $\mathrm{S}$ or UV and new medium was added. After 3-5 days of further culture, when untreated control cultures were still far from confluence, cells were pulse-labelled with [methyl ${ }^{3} \mathrm{H}$ ]-thymidine $(40-60 \mathrm{Ci} / \mathrm{mmol}, 5 \mu \mathrm{Ci} / \mathrm{ml})$ for $3 \mathrm{~h}$ in presence of $20 \mathrm{mM}$ HEPES $\mathrm{pH} 7.3$, to obtain a measure for the number of proliferating cells in each culture. After $30 \mathrm{~min}$ chase in unlabelled medium, cells were rinsed, lysed in $0.25 \mathrm{M}$ $\mathrm{NaOH}$ and harvested. The incorporated radioactivity, measured in a liquid scintillation counter, from four control dishes and duplicate dishes for each exposure were processed into mean \pm S.E. points. As reported earlier for human fibroblasts $[13,14]$ as well as rodent cells [11,15], this simplified protocol produces highly reproducible UV-survival curves covering three logs, and indistinguishable from those obtained with classical colony assays. With a few standard strains this was confirmed for illudin. Almost all presented panels with survival curves are from single representative experiments, containing negative and positive controls to ensure reproducibility. Error bars represent variability from triplicate of duplicate dishes.

Chinese hamster cells were sparsely seeded in dishes, exposed to various concentrations of illudin and allowed to form colonies. Chicken lymphoma cells were seeded at $10^{6}$ cells per $\mathrm{ml}$, exposed to illudin for $24-48 \mathrm{~h}$ and counted or checked for thymidine incorporation as above, immediately or after 1 day.

\subsection{Unscheduled DNA synthesis}

Coverslip cultures were exposed to $16 \mathrm{~J} / \mathrm{m}^{2}$ of UV light and cultured for $2 \mathrm{~h}$ in tritiated thymidine as described [16]. In case of illudin $S$, cells were incubated with $60 \mathrm{ng} / \mathrm{ml}$ in presence of [methyl, $\left.1^{\prime}, 2^{\prime}-{ }^{3} \mathrm{H}\right]$-thymidine $(120 \mathrm{Ci} / \mathrm{mmol}, 5 \mu \mathrm{Ci} / \mathrm{ml})$ for $24 \mathrm{~h}$. After radioactive labelling, all cultures were fixed and processed for autoradiography as described 
[16], and silver grains were counted over at least 50 non-S-phase cells.

\subsection{RNA synthesis inhibition and recovery}

One-day-old fibroblast cultures in $3 \mathrm{~cm}$-dishes were prelabelled with $\left[2-{ }^{14} \mathrm{C}\right]$-thymidine $(50 \mathrm{mCi} / \mathrm{mmol}$, $50 \mathrm{nCi} / \mathrm{ml}$ ) for $16 \mathrm{~h}$, exposed to illudin $\mathrm{S}$ for $3 \mathrm{~h}$ and cultured further. At various times, cells were pulse-labelled $(1 \mathrm{~h})$ with $\left[5,6-{ }^{3} \mathrm{H}\right]$-uridine (38 Ci $/ \mathrm{mmol}, 2 \mu \mathrm{Ci} / \mathrm{ml}$ ), alkali-lysed and processed for scintillation counting as described above. The ratios of ${ }^{3} \mathrm{H}$ to ${ }^{14} \mathrm{C}$ are taken as a measure of overall RNA synthesis [13].
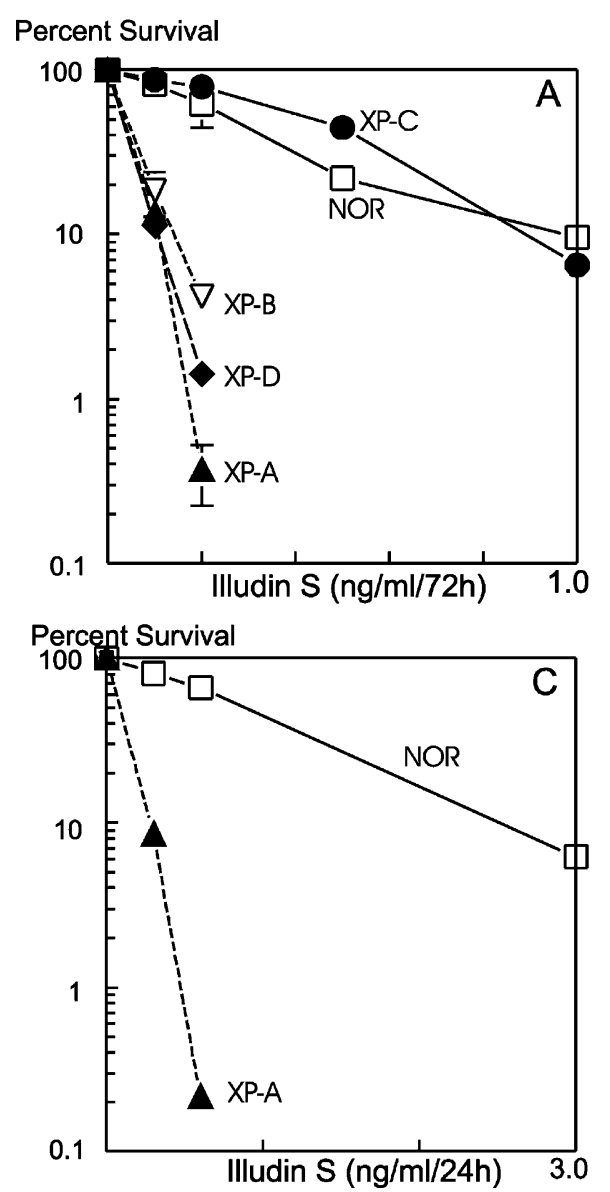

\section{Results}

\subsection{Core NER factors are required for repair of illudin damage}

Based on earlier experience with SV40-transformed human fibroblasts [9] a treatment protocol of $72 \mathrm{~h}$ with doses up to $1 \mathrm{ng} / \mathrm{ml}(0.26 \mathrm{nM})$ of illudin $\mathrm{S}$ was chosen for the primary fibroblasts. In these conditions, survival decreased exponentially with dose, revealing a $D_{10}$ value of about $1.1 \mathrm{ng} / \mathrm{ml}$ in normal control cells and $0.12 \mathrm{ng} / \mathrm{ml}$ for fully NER-deficient XP-A fibroblasts (see Fig. 1A). Thus, normal cytotoxicity was slightly higher than observed in a single
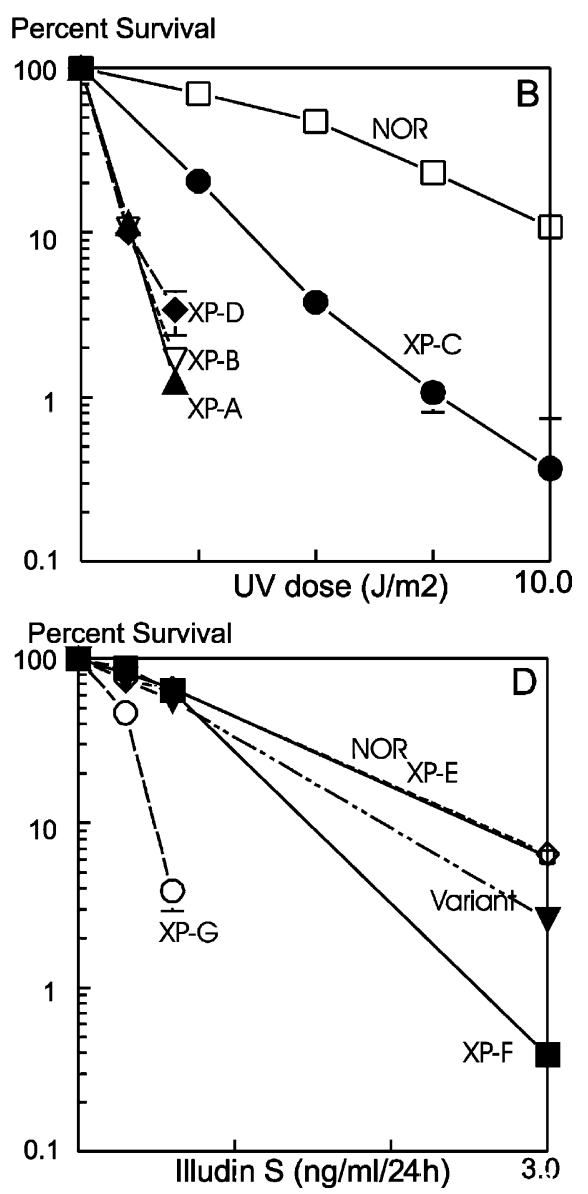

Fig. 1. Illudin S and UV sensitivities of xeroderma pigmentosum strains. Cells were exposed to graded doses of UV (panel (B)) or illudin $S$ (panel (A) for $72 \mathrm{~h}$-exposures and panel $(\mathrm{C}$ and $\mathrm{D})$ for $24 \mathrm{~h}$-exposures) and assayed for cell survival as indicated in the Section 2. Symbols:

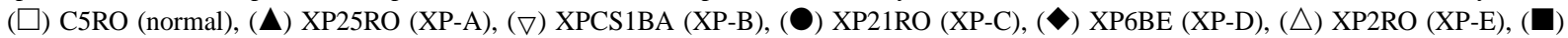
XP42RO (XP-F), (O) XP2BI (XP-G), ( $)$ XP1RO (variant). Error bars represent S.E. and are drawn wherever they exceed the symbol size. 
SV40-transformed normal human fibroblast culture [9]. The XP-A hypersensitivity ratio of $8-10$ was about the same as that obtained for UV-exposure, with $D_{10}$ values of about 1 and $10 \mathrm{~J} / \mathrm{m}^{2}$ for XP-A and normal cells, respectively (Fig. 1B). With shorter exposure times, used in later experiments, higher doses were required to produce the same lethality. An inverse relationship between time and dose was found to exist between concentrations of 0.5 and $10 \mathrm{ng} / \mathrm{ml}$ at least, with no significant change in relative sensitivity of NER-defective XP-cells (Figs. 1A and C and 3A and $\mathrm{B}$, and data not shown).

In UV-sensitive fibroblasts with defective XPB, XPD, XPF and XPG, other core enzymes of the NER-pathway, the responses to illudin $S$ were similarly exaggerated, though less pronounced. In all these cases, the hypersensitivity factors after exposure to illudin $\mathrm{S}$ and $\mathrm{UV}$ irradiation were comparable (Fig. $1 \mathrm{~A}, \mathrm{~B}$ and D), confirming the requirement of NER for removal of illudin lesions, and suggesting similar mechanisms of cytotoxicity.

\subsection{Global-genome NER is dispensable}

Unexpectedly, cells from XP group C, presented a clear exception: an intermediate sensitivity to UV (2.5-5) times (Fig. 1B), typical of XP-C strains [17] contrasted with a fully normal survival after illudin $\mathrm{S}$ exposure, a response found in three different human XP-C strains (Fig. 2A). In XPC-defective mouse fibroblasts, known to be sensitive to UV as well [18] this behaviour was confirmed (Fig. 2D). The possibility existed that normal XP-C survival was simply related to the protracted low dose treatment protocol in combination with residual repair activity. This explanation is highly unlikely since moderately UV-sensitive XP-F cells with a residual NER level of 15-30\% (similar to XP-C), were as sensitive to illudin as they were to UV (Fig. 1D). In order to rigorously exclude potential dosimetry effects, we confirmed $\mathrm{XP}-\mathrm{C}$ normalcy in a shorter $(24 \mathrm{~h})$ treatment protocol (Fig. 2B). Furthermore, both XP-A and XP-C cells retained their sensitivity to $\mathrm{UV}$, when irradiation was split in seven doses delivered over a $72 \mathrm{~h}$ period, in order to mimic a low-dose-rate regimen similar to illudin S (Fig. 2C). Consistent with the unexpected behaviour of XP-C cells, UV-sensitive mouse fibroblasts defective in both HR23A and HR23B proteins, which can bind XPC protein and stabilise it (Ng et al., manuscript in preparation), were also as resistant to illudin as normal mouse cells (Fig. 2D). In addition to XP-C cells, also XP-E cells survived illudin exposure normally (see Fig. 1D).

\subsection{Illudin lesions are exclusively removed by transcription-coupled NER}

The enzyme complexes containing XPC and XPE are responsible for the lesion-recognising steps in the NER subpathway which repairs global DNA damage. In XPE and XPC mutants preferential repair of actively transcribed DNA strands is fully intact. Consistent deficiency (to varying degrees) in both global-genome repair (GG-NER) and transcription-coupled repair (TC-NER) is a characteristic feature of the other XP groups. (for a review, see [19]). Our data suggest that only TC-NER is relevant to illudin cytotoxicity. Therefore, cells from CS patients, known to have a selective defect in TC-NER, were studied. CS-A and CS-B cells were seven and nine times more sensitive to illudin $\mathrm{S}$ than two cell strains with normal NER (see Fig. 3A). These responses to illudin are much more pronounced than to UV (two and four times, respectively, data not shown here. See $[13,17])$ and more near to fully NER-deficient XP-A cells (see Fig. 3C). We found exactly the same hypersensitivity pattern in cells from patients with UV-sensitivity syndrome (UVS, [20,21] see Fig. 3C), which also suffer from defective TC-NER [22,23].

TC-NER is initiated at transcription forks stalled at DNA lesions and allows completion of the transcript after removal of the lesion (for overview see: [24]). To study the inhibition and recovery of RNA synthesis, the fibroblasts were exposed to illudin in a shortened, $3 \mathrm{~h}$-exposure protocol, followed by a pulse-labelling with tritiated uridine at various times (Fig. 3B). Initial inhibition and subsequent recovery were evident in normal and XP-C cells, but did not at all occur in fully NER-defective XP group A. We conclude, that illudin S-induced lesions indeed inhibit transcription and require TC-NER to overcome this.

To investigate the actual rates of NER, we measured illudin S-induced repair DNA synthesis by autoradiography (unscheduled DNA synthesis (UDS)). Even in normal cells we found UDS to be very low: only 

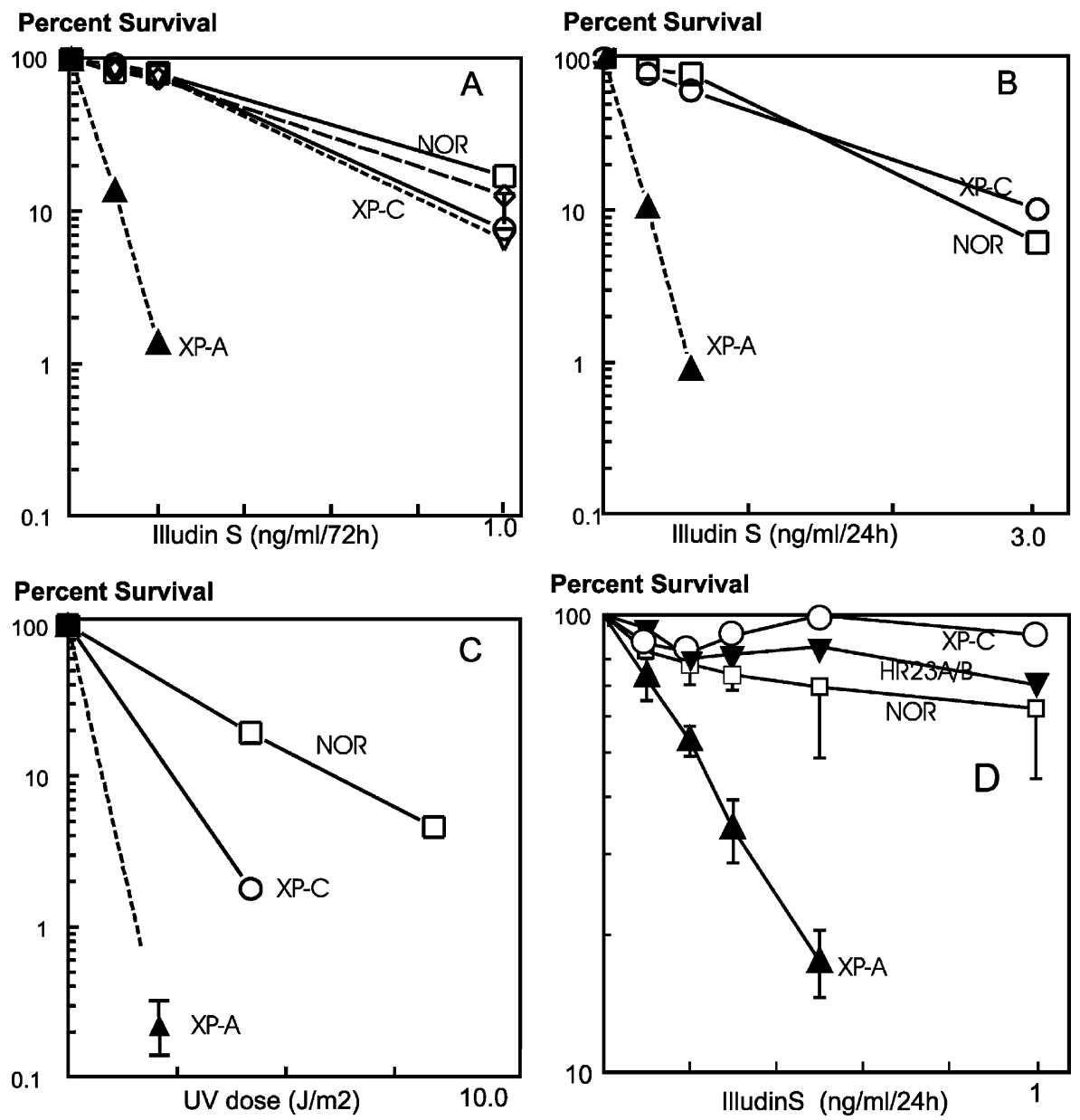

Fig. 2. XPC/HR23 complex is dispensable for illudin survival. Survival curves obtained as in Fig. 1 after exposure to illudin S (panel (A) for $72 \mathrm{~h}$ and panels (B and D) for $24 \mathrm{~h}$ ) or to UV (panel C). Panel (A): three different XP-C strains tested against XP-A and normal cells. Panel (B): XP-C survival after a $24 \mathrm{~h}$ exposure to illudin. Panel (C): extended UV-exposure protocol mimicking low dose rate; cells were irradiated six times over a period of $72 \mathrm{~h}$, summed up to the total doses indicated. Panel (D): illudin sensitivity of fibroblasts from KO

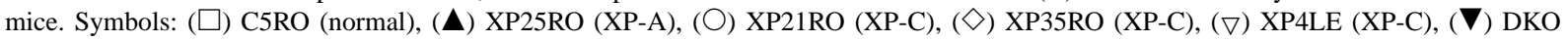
(HR23A/HR23B double mutant). Error bars represent S.E. and drawn wherever they exceed the symbol size.

after an exposure to relatively high doses $(60 \mathrm{ng} / \mathrm{ml}$ for $24 \mathrm{~h}$, about 20 times higher than the $D_{10}$ equivalent) and an extended radioactivity incorporation period during exposure (eight times longer than usually applied with UV), significant UDS above background could be measured in normal fibroblasts. Calculations showed these levels to be at least 30 times lower than what is common for a saturating dose of UV $\left(16 \mathrm{~J} / \mathrm{m}^{2}\right)$, consistent with the estimated low levels of lesions [11]. The UDS we observed clearly represented NER, since it was absent in XP-A cells (Fig. 3E). Despite their defective GG-NER, XP-C cells showed approximately the same level as normal cells, whereas in CS cells, carrying the complementary defect in TC-NER, only marginal levels of UDS were detected, comparable to XP-A cells (Fig. 3E). This result was found in both CS-A and CS-B and strongly contrasts with the characteristic normal UDS levels after UV exposure in these strains (see Fig. 3D and E) [17]. We conclude that the observed (low) UDS activity of normal fibroblasts exclusively represents TC-NER, with no significant contribution of GG-NER. 


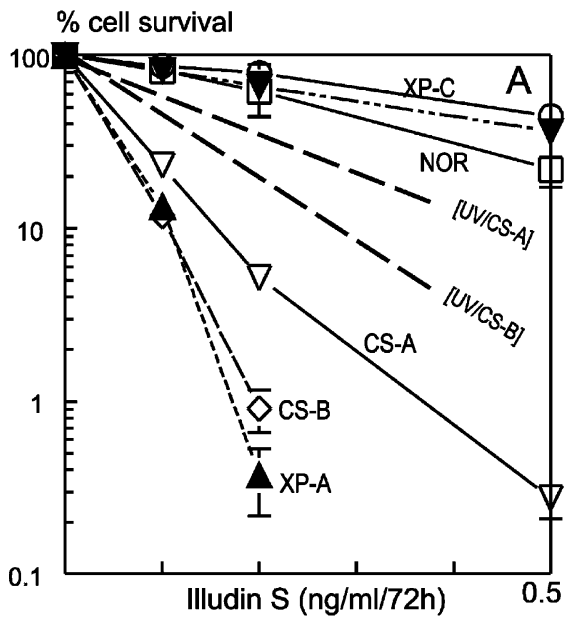

\section{$\%$ RNA synthesis (treated/untreated)}

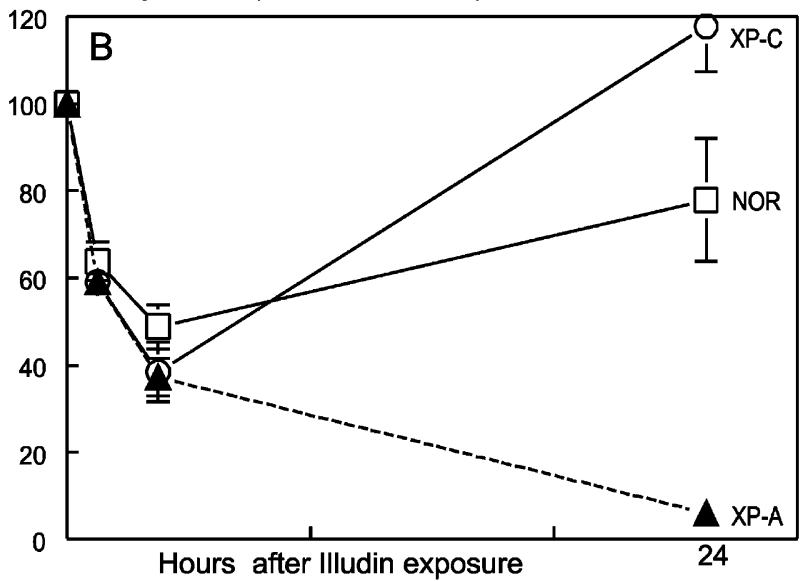

$\%$ cell survival
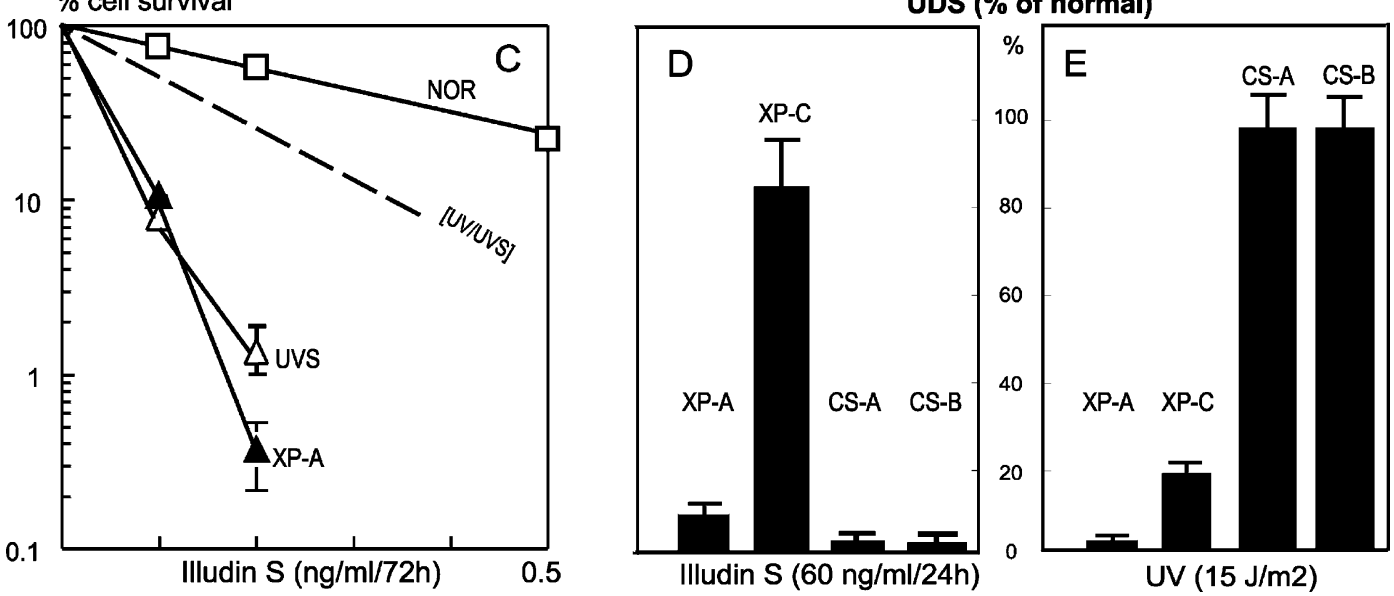

Fig. 3. Illudin survival depends on transcription-coupled NER. Panels (A and C): sensitivity of CS cells (panel (A)) and UVS (panel (C)) to illudin S. For comparison, dashed lines indicate the UV-sensitivities obtained with these cell strains, on a modified dose scale, such that normal response curves of UV and illudin overlap. Panel (B): recovery of overall transcription after illudin exposure. Cells were prelabelled with ${ }^{14} \mathrm{C}$-thymidine, exposed to 0 or $30 \mathrm{ng} / \mathrm{ml}$ of illudin $\mathrm{S}$ for $3 \mathrm{~h}$, rinsed and pulse-labelled with ${ }^{3} \mathrm{H}$-uridine 1,3 and $21 \mathrm{~h}$ later. Relative rates of RNA synthesis are expressed as percentages of treated over mock-treated cells. Symbols in panels (A-C): ( $\square$ ) C5RO (normal),

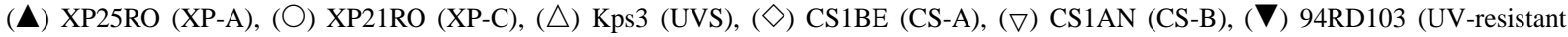
trichothiodystrophy). Panels (D and E): unscheduled DNA synthesis induced by illudin S and UV, respectively. Averaged data plotted as a percentage of responses in normal cells and compared to similarly plotted UDS-levels induced by UV.

Hydroxymethylacylfulvene (HMAF, Irofulven or MGI-114) is a semisynthetic derivative of illudin S [25], which is currently under clinical trial for tumour therapy [4]. Fig. 4 shows that this agent is about 50 times less toxic to human fibroblasts than illudin $\mathrm{S}$ (calculated $D_{10}$ values 50 and $1 \mathrm{ng} / \mathrm{ml}$ per $72 \mathrm{~h}$, respectively). However, a 10 times increased sensitivity in XP-A cells, combined with normal XP-C responses were also found with HMAF (Fig. 4), suggesting that this drug acts via the same mechanism as the other illudins.

\subsection{Involvement of other global repair pathways}

If global illudin damage is not processed via the NER pathway, the question remains whether other global-genome repair pathways can take over. Therefore we studied a number of Chinese hamster mutants 


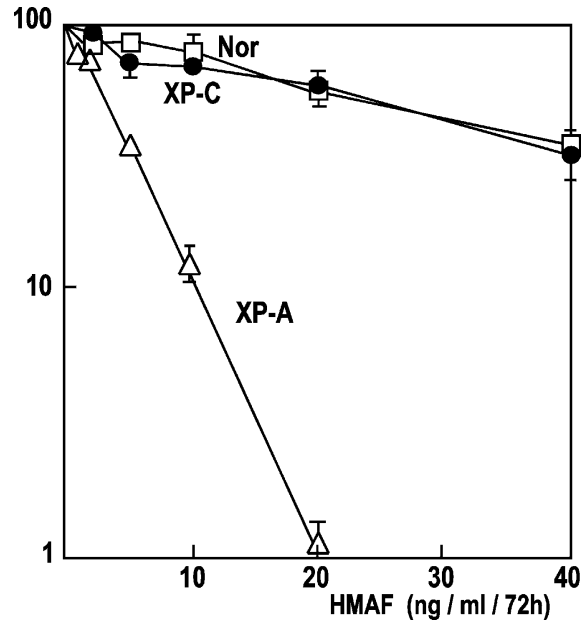

Fig. 4. Cytotoxicity of HMAF. Human fibroblast survival curves as determined in Fig. 1 after exposure to HMAF (Irofulven, MGI-114). Symbols: ( $\triangle$ ) XP25RO (XP-A), (O) XP21RO (XP-C), ( $\square$ ) C5RO (normal). with known defects in BER and NHEJ pathways (all listed in Table 1). In colony-survival assays, XRCC1-mutant BER-deficient cells as well as three different NHEJ mutants (Ku86, DNA-PKcs, XRCC4) were all as sensitive to illudin as their parental $\mathrm{CHO}$ strains and/or mutant strains corrected with a normal copy of their defective gene (data not shown). This resistance is not specific to $\mathrm{CHO}$ cell metabolism, since NER-deficient $\mathrm{CHO}$ mutants are hypersensitive [11]. We conclude, that the two globally acting repair pathways BER and NHEJ play no significant role in illudin cytotoxicity. These data also suggest that illudin lesions do not cause significant levels of DNA breakage.

\subsection{Illudin lesions presumably obstruct replication forks}

We have shown earlier, that in cells exposed to illudin S, DNA synthesis is inhibited [2]. To investigate the mechanism of DNA-replication interference we measured the requirement of genes involved in post-replication repair (PRR) pathways. In yeast
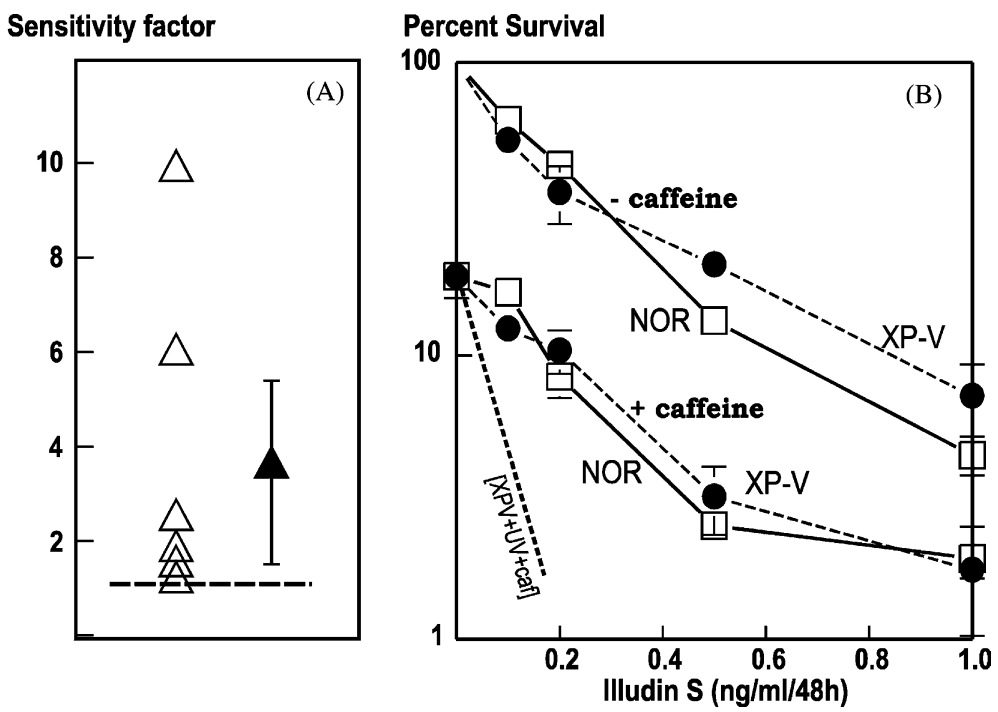

Fig. 5. Post-replication repair and cytotoxicity of illudin S. Panel (A): RAD18-deficient chicken lymphoma cells. Open symbols $(\triangle$ ) represent mutant/WT sensitivity ratios obtained in seven separate survival experiments, under somewhat varying conditions (cell density, time of treatment, etc). Closed symbol $(\boldsymbol{\Delta})$ represents the mean \pm S.D. of these tests. Panel (B): human XP-variant and normal fibroblasts, exposed to illudin in absence or presence of $1.5 \mathrm{mM}$ caffeine and cultured further ( \pm caffeine) for survival determination. Curves are normalised to values without caffeine, to indicate aspecific toxicity of caffeine (lower curves). The dashed line without symbols represents sensitisation response obtained with XP-variant cells exposed to UV and caffeine (from a separate experiment). Symbols: ( $\square$ ) C5RO (normal), (O) XP30RO (XP-variant). 
as well as human cells the RAD18/RAD6 protein complex is involved in an early step of PRR [26-28]. Chicken DT40 lymphoma cells with two defective RAD18 alleles are about two times hypersensitive to UV (Yamashita et al., manuscript in preparation). For unknown reasons, chicken lymphoma cells responded to illudin in a highly variable manner, irrespective of the different standardised growth conditions tested. Fig. 5A summarises all our data, indicating that RAD18-deficient cells are hypersensitive to illudin, although experimental variability does not allow a precise estimation of the extent.

Polymerase $\eta$ (RAD30A) is one of the translesion polymerases active in a downstream branch of the PRR pathway (for overviews see: $[29,30]$ ). Absence of this polymerase in XP-variant fibroblasts [31] did not or hardly affect illudin toxicity (see Fig. 1D and 5B) Sensitisation by caffeine, which is a characteristic feature of UV-irradiated pol $\eta$ mutants $[32,33]$ was also not observed with illudin (Fig. 5B). It follows that the downstream translesion subpathway of PRR is either not required or is accomplished by another translesion polymerase (e.g. pol ı, pol $\zeta$ [34]) which we could not investigate due to lack of mutants.

\section{Discussion}

Our present detailed data with cell strains from patients having xeroderma pigmentosum and from mice with targeted NER mutations, confirm and extend an early observation in CHO-cells, that NER can repair illudin lesions $[11,35]$. The overall responses of the non-transformed fibroblasts are highly reproducible over different experiments and cell strains and are found in the same order of magnitude as in relatively resistant tumour cells in culture [9] which appear to rule out large differences in transport and/or metabolic capacities.

Total absence of NER (as occurring in XP-A cells) renders human and mouse fibroblasts about 10 times more sensitive to illudin $\mathrm{S}$, the same enhancement as seen with exposure to UV. This parallelism is retained in other XP complementation groups with NER defects of varying severity. Unexpectedly however, cells defective in components of the HR23/XPC complex, present a notable exception, being normally resistant to the drug. We conclude, that repair factor
$\mathrm{XPC} / \mathrm{HR} 23$ is not used or not required for NER of illudin-induced lesions. The XPC/HR23B protein complex is known to be selectively involved in global-genome repair (GG-NER) [36], where it performs an early function in lesion recognition $[37,38]$ and recruitment of subsequent core NER enzymes [39]. Dispensability of GG-NER was further supported by the independence of XPE, a gene encoding a component of the DDB complex, which plays a complementary role in initiation of GG-NER [40].

According to current models [19,24,41] transcription forks stalled at a lesion in the transcribed strand form a structure that can initiate the so-called transcription-coupled subpathway of NER (TC-NER) without the need of damage recognition by the XPC/HR23B and/or DDB complexes, but still requiring all the other NER factors. We find here that illudin lesions do interfere with transcription, and require TC-NER to recover. Cells defective in specific TC-NER factors (CSA,CSB,UVS) are more sensitive to illudin then they are to UV. The low levels of overall repair (measured as UDS) fully depend on these TC-NER factors. Unfortunately, these low levels and their prolonged induction times have hampered our attempts to perform actual biochemical measurements of transcribed strand-directed repair of actively expressed genes. Since in general, the rate of overall removal of lesions by NER largely depends on the affinity and levels of HR23/XPC [38] and DDB complexes [42], our data are best compatible with the notion that illudin lesions are recognised by GG-NER with very low, undetectable affinity.

While NERable lesions appear to constitute about $90 \%$ of potentially lethal damage in fibroblasts, actively transcribed regions represent a much smaller fraction of the genome. It appears that prolonged stalling of transcription forks are the primary cytotoxic entity initiating the apoptotic sequence, as suggested on other grounds earlier [43-45]. Therefore, alternative or additional routes for global repair of illudin lesions could also contribute to protection against illudin. Our data with XRCC1-defective cells indicate that the most common short-patch branch of base excision repair $[46,47]$ is probably not a candidate. The possibility that illudin-lesions lead to significant levels of strand breakage is also considered unlikely, since non-homologous end-joining defects confer no sensitivity to illudin. Taken together, our 
data strongly suggest that illudin-lesions are ignored by all of the known global repair pathways.

Illudin damage not only affects transcription complexes, but inhibit DNA replication as well [2]. We show that there is a strong requirement for RAD18, to overcome such replication blockage. Chicken cells had to be studied here, being the only RAD18-deficient cells currently available. In parallel with TC-NER defective cells (CS-A, CS-B, UVS), they are more sensitive to illudin than to UV. The RAD18/RAD6 protein complex accomplishes an early step in post-replication repair (PRR) [26-28]. A downstream subpathway of PRR involves translesion synthesis by one of a range of recently identified tolerant DNA polymerases [30]. The fully normal responses of XP-variant cells show that polymerase $\eta$ (with preference for UV CPD-lesions [48,49]) is not the enzyme responsible for this action. However, the involvement of other candidate translesion polymerases (e.g. pol $\zeta$, pol ı) with different specificities [50] was not investigated here yet. A role in illudin cytotoxicity of the alternative RAD5-dependent downstream subpathway of PRR [51] also remains to be checked. Finally, we do not yet know whether there is involvement of other replication-coupled repair modes, such as recombination or mismatch-repair, in recovery from illudin lethality. However, especially with respect to recombinational repair such involvement seems likely, since prolonged stalling of replication forks (e.g. in RAD18-defective cells) is known to increase the frequency of recombination [52]. Moreover, topoisomerase inhibitors, expected to compromise recombinational processing of stalled replication forks, were shown to sensitise cells to illudins $[25,53,54]$.

Information on the nature, chemical structure and stability of illudin-induced DNA lesions, is still lacking. Illudins probably do not produce intrastrand crosslinks, in view of moderate sensitivity of ERCC1-deficient cells ([11] and data not shown). Our data indicate that global repair pathways appear not to act on the lesion, which is therefore unlikely to cause major helix-distortion. To our knowledge, such an unusual repair pattern has so far only been found with a new class of agents producing adducts exclusively in the minor groove of the DNA helix [55]. The susceptibility to TC-NER was not investigated by these authors, but the exquisite sensitisation in RAD18 mutants which they observed, is fully consistent with our data. Ecteinascidine 743 offers another case of TC-NER-specificity for minor-groove alkylation [56], although this lesion seems to poison the NER process itself.

In conclusion, we show here that illudins, belonging to a new and promising class of tumour-therapeutic agents, produce a unique type of DNA lesion which is largely ignored by global repair pathways. Only when the lesions become trapped in transcription-complexes or replication-forks, efficient repair mechanisms ensue, such as TC-NER or PRR. As a consequence, we find that gene defects involved in these transcriptionand replication-coupled repair pathways confer highly exaggerated sensitivity to the illudins, in comparison to, e.g. UV light. Further studies on more complicated endpoints such as mutagenesis and responses in repair-deficient mice will be needed to expand on this issue.

\section{Acknowledgements}

The authors wish to thank Drs. M.Z. Zdzienicka and M. Modesti for mutated/corrected $\mathrm{CHO}$ clones, Dr. M. Yamaizumi for allowing us the use of Kps3 (UVS) cells and Dr. L.F.M. Mullenders for comments. This research was partially supported by the CEC Concerted Action Programme on DNA Repair and by TRDRP project grant 9RT-0057 to MJK.

\section{References}

[1] T.C. McMorris, M. Anchel, Fungal metabolites. The structures of the novel sesquiterpenes illudin S and M, J. Am. Chem. Soc. 87 (1965) 1594-1600.

[2] M.J. Kelner, T.C. McMorris, W.T. Beck, J.M. Zamora, R. Taetle, Preclinical evaluation of illudins as anticancer agents, Cancer Res. 47 (1987) 3186-3189.

[3] M.J. Kelner, T.C. McMorris, M.A. Montoya, L. Estes, M. Rutherford, K.M. Samson, R. Taetle, Characterization of cellular accumulation and toxicity of illudin $\mathrm{S}$ in sensitive and nonsensitive tumor cells, Cancer Chemother. Pharmacol. 40 (1997) 65-71.

[4] T.C. McMorris, Discovery and development of sesquiterpenoid derived hydroxymethylacylfulvene: a new anticancer drug, Bioorg. Med. Chem. 7 (1999) 881-886.

[5] J.R. MacDonald, C.C. Muscoplat, D.L. Dexter, G.L. Mangold, S.F. Chen, M.J. Kelner, T.C. McMorris, D.D. Von Hoff, Preclinical antitumor activity of 6-hydroxymethylacylfulvene, a semisynthetic derivative of the mushroom toxin illudin S, Cancer Res. 57 (1997) 279-283. 
[6] Y. Sato, S. Kashimoto, J.R. MacDonald, K. Nakano, In vivo antitumour efficacy of MGI-114 (6-hydroxymethylacylfulvene HMAF) in various human tumour xenograft models including several lung and gastric tumours, Eur. J. Cancer 37 (2001) $1419-1428$.

[7] J.P. Thomas, R. Arzoomanian, D. Alberti, C. Feierabend, K. Binger, K.D. Tutsch, T. Steele, R. Marnocha, C. Smith, S. Smith, J. MacDonald, G. Wilding, H. Bailey, Phase I clinical and pharmacokinetic trial of Irofulven, Cancer Chemother. Pharmacol. 48 (2001) 467-472.

[8] F. Giles, J. Cortes, G. Garcia-Manero, S. Kornblau, E. Estey, M. Kwari, A. Murgo, H. Kantarjian, Phase I study of Irofulven (MGI-114), an acylfulvene illudin analog, in patients with acute leukemia, Invest. New Drugs 19 (2001) 13-20.

[9] M.J. Kelner, T.C. McMorris, R. Taetle, Preclinical evaluation of illudins as anticancer agents: basis for selective cytotoxicity, J. Natl. Cancer Inst. 82 (1990) 1562-1565.

[10] K. Tanaka, T. Inoue, S. Kadota, T. Kikuchi, Metabolism of illudin S, a toxic principle of Lampteromyces japonicus, by rat liver. I. Isolation and identification of cyclopropane ring-cleavage metabolites, Xenobiotica 20 (1990) 671-681.

[11] M.J. Kelner, T.C. McMorris, L. Estes, M. Rutherford, M. Montoya, J. Goldstein, K. Samson, R. Starr, R. Taetle, Characterization of illudin $\mathrm{S}$ sensitivity in DNA repair-deficient Chinese hamster cells. Unusually high sensitivity of ERCC2 and ERCC3 DNA helicase-deficient mutants in comparison to other chemotherapeutic agents, Biochem. Pharmacol. 48 (1994) 403-409.

[12] M. Anchel, A. Hervey, W. Robbins, Antibiotic substances from basidiomycetes. VII. Clitocybe illudins, Proc. Natl. Acad. Sci. U.S.A. 36 (1950) 30-36.

[13] B.C. Hamel, A. Raams, A.R. Schuitema-Dijkstra, P. Simons, I. van der Burgt, N.G.J. Jaspers, W.J. Kleijer, Xeroderma pigmentosum-Cockayne syndrome complex: a further case, J. Med. Genet. 33 (1996) 607-610.

[14] A.M. Sijbers, P.C. van Voorst Vader, J.W. Snoek, A. Raams, N.G.J. Jaspers, W.J. Kleijer, Homozygous R788W point mutation in the $X P F$ gene of a patient with xeroderma pigmentosum and late-onset neurologic disease, J. Invest. Dermatol. 110 (1998) 832-836.

[15] A.M. Sijbers, P.J. van der Spek, H. Odijk, J. van den Berg, M. van Duin, A. Westerveld, N.G.J. Jaspers, D. Bootsma, J.H. Hoeijmakers, Mutational analysis of the human nucleotide excision repair gene ERCC1, Nucleic Acids Res. 24 (1996) 3370-3380.

[16] W. Vermeulen, R.J. Scott, S. Rodgers, H.J. Muller, J. Cole, C.F. Arlett, W.J. Kleijer, D. Bootsma, J.H. Hoeijmakers, G. Weeda, Clinical heterogeneity within xeroderma pigmentosum associated with mutations in the DNA repair and transcription gene ERCC3, Am. J. Hum. Genet. 54 (1994) 191-200.

[17] D. Bootsma, K. Kraemer, J. Cleaver, J. Hoeijmakers, Nucleotide excision repair syndromes: xeroderma pigmentosum, Cockayne syndrome and trichothiodystrophy, in: C. Scriver, A. Beaudet, W. Sly, D. Valle (Eds.), The Metabolic Basis of Inherited Disease, McGraw-Hill, New York, 1997, p. 159.

[18] D.L. Cheo, H.J. Ruven, L.B. Meira, R.E. Hammer, D.K. Burns, N.J. Tappe, A.A. van Zeeland, L.H. Mullenders, E.C.
Friedberg, Characterization of defective nucleotide excision repair in XPC mutant mice, Mutat. Res. 374 (1997) 1-9.

[19] W.L. de Laat, N.G.J. Jaspers, N.G.J. Hoeijmakers, Molecular mechanism of nucleotide excision repair, Genes Dev. 13 (1999) 768-785.

[20] T. Itoh, T. Ono, M. Yamaizumi, A new UV-sensitive syndrome not belonging to any complementation groups of xeroderma pigmentosum or Cockayne syndrome: siblings showing biochemical characteristics of Cockayne syndrome without typical clinical manifestations, Mutat. Res. 314 (1994) 233-248.

[21] T. Itoh, M. Yamaizumi, M. Ichihashi, M. Hiro-Oka, T. Matsui, M. Matsuno, T. Ono, Clinical characteristics of three patients with UVS syndrome, a photosensitive disorder with defective DNA repair, Br. J. Dermatol. 134 (1996) 1147-1150.

[22] T. Itoh, M. Yamaizumi, UVS syndrome: establishment and characterization of fibroblastic cell lines transformed with simian virus 40 DNA, J. Invest. Dermatol. 114 (2000) 101106.

[23] G. Spivak, T. Itoh, P. Hanawalt, M. Yamaizumi, UV-sensitive syndrome cells have a defect in transcription-coupled repair of UV-induced cyclobutane pryimidine dimers, in: Proceedings of the Workshop on DNA Repair: Interplay with Other Cellular Processes, Noordwijkerhout, The Netherlands, 2001, p. 123 (Abstract).

[24] A.J. van Gool, G.T. van der Horst, E. Citterio, J.H. Hoeijmakers, Cockayne syndrome: defective repair of transcription? EMBO J. 16 (1997) 4155-4162.

[25] M.J. Kelner, T.C. McMorris, L. Estes, K.M. Samson, N.A. Trani, J.R. MacDonald, Anti-leukemic action of the novel agent MGI-114 (HMAF) and synergistic action with topotecan, Leukemia 14 (2000) 136-141.

[26] S. Tateishi, Y. Sakuraba, S. Masuyama, H. Inoue, M. Yamaizumi, Dysfunction of human RAD18 results in defective post-replication repair and hypersensitivity to multiple mutagens, Proc. Natl. Acad. Sci. U.S.A. 97 (2000) 79277932.

[27] R. van der Laan, H.P. Roest, J.W. Hoogerbrugge, E.M. Smit, R. Slater, W.M. Baarends, J.H. Hoeijmakers, J.A. Grootegoed, Characterization of $m R A D 18 S c$, a mouse homolog of the yeast post-replication repair gene $R A D 18$, Genomics 69 (2000) 8694.

[28] S. Broomfield, T. Hryciw, W. Xiao, DNA post-replication repair and mutagenesis in Saccharomyces cerevisiae, Mutat. Res. 486 (2001) 167-184.

[29] H. Ohmori, E.C. Friedberg, R.P. Fuchs, M.F. Goodman, F. Hanaoka, D. Hinkle, T.A. Kunkel, C.W. Lawrence, Z. Livneh, T. Nohmi, L. Prakash, S. Prakash, T. Todo, G.C. Walker, Z. Wang, R. Woodgate, The Y-family of DNA polymerases, Mol. Cell 8 (2001) 7-8.

[30] E.C. Friedberg, P.L. Fischhaber, C. Kisker, Error-prone DNA polymerases: novel structures and the benefits of infidelity, Cell 107 (2001) 9-12.

[31] C. Masutani, R. Kusumoto, A. Yamada, N. Dohmae, M. Yokoi, M. Yuasa, M. Araki, S. Iwai, K. Takio, F. Hanaoka, The XPV (xeroderma pigmentosum variant) gene encodes human DNA polymerase eta, Nature 399 (1999) 700-704. 
[32] B.C. Broughton, A. Cordonnier, W.J. Kleijer, N.G. Jaspers, H. Fawcett, A. Raams, V.H. Garritsen, A. Stary, M.F. Avril, F. Boudsocq, C. Masutani, F. Hanaoka, R.P. Fuchs, A. Sarasin, A.R. Lehmann, Molecular analysis of mutations in DNA polymerase eta in xeroderma pigmentosum-variant patients, Proc. Natl. Acad. Sci. U.S.A. 99 (2002) 815-820.

[33] T. Itoh, S. Linn, R. Kamide, H. Tokushige, N. Katori, Y. Hosaka, M. Yamaizumi, Xeroderma pigmentosum variant heterozygotes show reduced levels of recovery of replicative DNA synthesis in the presence of caffeine after ultraviolet irradiation, J. Invest. Dermatol. 115 (2000) 981-985.

[34] J.P. McDonald, A. Tissier, E.G. Frank, S. Iwai, F. Hanaoka, R. Woodgate, DNA polymerase iota and related rad30-like enzymes, Philos. Trans. R. Soc. Lond. B Biol. Sci. 356 (2001) 53-60.

[35] M.J. Kelner, T.C. McMorris, L. Estes, R.J. Starr, M. Rutherford, M. Montoya, K.M. Samson, R. Taetle, Efficacy of acylfulvene illudin analogues against a metastatic lung carcinoma MV522 xenograft nonresponsive to traditional anticancer agents: retention of activity against various $\mathrm{mdr}$ phenotypes and unusual cytotoxicity against ERCC2 and ERCC3 DNA helicase-deficient cells, Cancer Res. 55 (1995) 4490-4936.

[36] J. Venema, L.H. Mullenders, A.T. Natarajan, A.A. van Zeeland, L.V. Mayne, The genetic defect in Cockayne syndrome is associated with a defect in repair of UV-induced DNA damage in transcriptionally active DNA, Proc. Natl. Acad. Sci. U.S.A. 87 (1990) 4707-4711.

[37] M. Wakasugi, A. Sancar, Assembly, subunit composition, and footprint of human DNA repair excision nuclease, Proc. Natl. Acad. Sci. U.S.A. 95 (1998) 6669-6674.

[38] K. Sugasawa, J.M. Ng, C. Masutani, S. Iwai, P.J. van der Spek, A.P. Eker, F. Hanaoka, D. Bootsma, J.H. Hoeijmakers, Xeroderma pigmentosum group $\mathrm{C}$ protein complex is the initiator of global-genome nucleotide excision repair, Mol. Cell 2 (1998) 223-232.

[39] M. Volker, M.J. Mone, P. Karmakar, A. van Hoffen, W. Schul, W. Vermeulen, J.H. Hoeijmakers, R. van Driel, A.A. van Zeeland, L.H. Mullenders, Sequential assembly of the nucleotide excision repair factors in vivo, Mol. Cell 8 (2001) 213-224.

[40] B.J. Hwang, J.M. Ford, P.C. Hanawalt, G. Chu, Expression of the p48 xeroderma pigmentosum gene is p53-dependent and is involved in global genomic repair, Proc. Natl. Acad. Sci. U.S.A. 96 (1999) 424-428.

[41] P.C. Hanawalt, Role of transcription-coupled DNA repair in susceptibility to environmental carcinogenesis, Environ. Health Perspect. 104 (1996) 547-551.

[42] M. Wakasugi, A. Kawashima, H. Morioka, S. Linn, A. Sancar, T. Mori, O. Nikaido, T. Matsunaga, DDB accumulates at DNA damage sites immediately after UV irradiation and directly stimulates nucleotide excision repair, J. Biol. Chem. 277 (2002) 1637-1640.

[43] M. Ljungman, F. Zhang, Blockage of RNA polymerase as a possible trigger for UV light-induced apoptosis, Oncogene 13 (1996) 823-831.
[44] M. Yamaizumi, T. Sugano, UV-induced nuclear accumulation of p53 is evoked through DNA damage of actively transcribed genes independent of the cell cycle, Oncogene 9 (1994) 27752784.

[45] G. Conforti, T. Nardo, M. D’Incalci, M. Stefanini, Proneness to UV-induced apoptosis in human fibroblasts defective in transcription-coupled repair is associated with lack of $\mathrm{mdm} 2$ transactivation, Oncogene 18 (2000) 2714-2720.

[46] A. Klungland, T. Lindahl, Second pathway for completion of human DNA base excision repair: reconstitution with purified proteins and requirement for DNase IV (FEN1), EMBO J. 16 (1997) 3341-3348.

[47] S. Mitra, I. Boldogh, T. Izumi, T.K. Hazra, Complexities of the DNA base excision repair pathway for repair of oxidative DNA damage. A review, Environ. Mol. Mutagen. 38 (2001) 180-190.

[48] N. Nikolaishvili-Feinberg, M. Cordeiro-Stone, Bypass replication in vitro of UV-induced photoproducts blocking leading or lagging strand synthesis, Biochemistry 40 (2001) $15215-15223$.

[49] S.L. Yu, R.E. Johnson, S. Prakash, L. Prakash, Requirement of DNA polymerase eta for error-free bypass of UV-induced CC and TC photoproducts, Mol. Cell. Biol. 21 (2001) 185188.

[50] Z. Wang, Translesion synthesis by the UmuC family of DNA polymerases, Mutat. Res. 486 (2001) 59-70.

[51] W. Xiao, B.L. Chow, S. Broomfield, M. Hanna, The Saccharomyces cerevisiae RAD6 group is composed of an error-prone and two error-free post-replication repair pathways, Genetics 155 (2000) 1633-1641.

[52] B. Liefshitz, R. Steinlauf, A. Friedl, F. Eckardt-Schupp, M. Kupiec, Genetic interactions between mutants of the error-prone repair group of Saccharomyces cerevisiae and their effect on recombination and mutagenesis, Mutat. Res. 407 (1998) 135-145.

[53] L.A. Hammond, S.G. Hilsenbeck, S.G. Eckhardt, J. Marty, G. Mangold, J.R. MacDonald, E.K. Rowinsky, D.D. Von Hoff, S. Weitman, Enhanced antitumour activity of 6-hydroxymethylacylfulvene in combination with topotecan or paclitaxel in the MV522 lung carcinoma xenograft model, Eur. J. Cancer 36 (2000) 2430-2436.

[54] S. Weitman, H. Barrera, R. Moore, C. Gonzalez, J. Marty, S. Hilsenbeck, J.R. MacDonald, S.J. Waters, D. Von Hoff, MGI-114: augmentation of antitumor activity when combined with topotecan, J. Pediatr. Hematol. Oncol. 22 (2000) 306314.

[55] N. Brooks, P.J. McHugh, M. Lee, J.A. Hartley, Alteration in the choice of DNA repair pathway with increasing sequence selective DNA alkylation in the minor groove, Chem. Biol. 7 (2000) 659-668.

[56] Y. Takebayashi, P. Pourquier, D.B. Zimonjic, K. Nakayama, S. Emmert, T. Ueda, Y. Urasaki, A. Kanzaki, S.I. Akiyama, N. Popescu, K.H. Kraemer, Y. Pommier, Antiproliferative activity of ecteinascidin 743 is dependent upon transcription-coupled nucleotide excision repair, Nat. Med. 7 (2001) 961-966. 